\title{
Indirekte Effekte von Abrufübungen - Intuitiv und doch häufig unterschätzt
}

\author{
Tino Endres $\mathbb{D} \cdot$ Alexander Renkl $\mathbb{D}$
}

Eingegangen: 1. April 2021 / Überarbeitet: 5. November 2021 / Angenommen: 4. Dezember 2021 /

Online publiziert: 23. Dezember 2021

(C) Der/die Autor(en) 2021

Zusammenfassung Aufgaben, die den Abruf von kürzlich gelernten Inhalten erfordern, können das Erinnern dieser Inhalte erleichtern (,,Testing Effekt“"). Abrufaufgaben können aber auch metakognitive, motivationale und kognitive Auswirkungen haben, die die Effizienz des weiteren Lernens nach dem Abruf beeinflussen. Diese Einflüsse werden als indirekte Effekte der Abrufübungen bezeichnet. Auch wenn diese indirekten Abrufeffekte selten isoliert untersucht wurden, gibt es bereits eine Vielzahl an Erkenntnissen aus verschiedenen Forschungstraditionen, die uns erlauben Rückschlüsse darauf zu ziehen, wie sich Abrufübungen auf das nachfolgende Lernen auswirken und welche Lernziele wie erreicht werden können. In diesem Artikel werden metakognitive, motivationale und lernzielspezifische Effekte zusammengestellt und erläutert, wie diese im Unterricht genutzt werden können. Dabei legen wir besonderes Augenmerk auf bedeutungshaltiges Lernen, das auf Verständnis des Lernstoffes abzielt. In unserer Zusammenstellung wird deutlich, dass es keine ideale Art gibt, indirekte Effekte von Abrufübungen zu nutzen. Je nach Lernzielen und den Eigenschaften der Lernenden sind unterschiedliche Arten der indirekten Abrufübungen zu empfehlen, da unterschiedliche Abrufarten jeweils Schwächen und Stärken in Bezug auf bestimmte Ziele haben. Sowohl Lehrende als auch Lernende sollten um die spezifischen Wirkmechanismen der indirekten Effekte der Abrufübungen wissen, um das eigene Lernverhalten oder das anderer Lernender gezielt optimieren zu können.

Schlüsselwörter Indirekte Testing-Effekte · Metakognitive Überwachung · Motivation · Lernzielspezifische Effekte

Tino Endres $(\bowtie) \cdot$ Alexander Renkl

Abteilung für Entwicklungs- und Pädagogische Psychologie, Albert-Ludwigs-Universität Freiburg,

Engelbergerstraße 41, 79085 Freiburg, Deutschland

E-Mail: tino.endres@psychologie.uni-freiburg.de 


\title{
Indirect effects of retrieval practice-Intuitive yet often underestimated.
}

\begin{abstract}
Learning tasks, that require the retrieval of recently learned contents can help remember these contents (testing effect). However, retrieval tasks can also have substantial metacognitive, motivational, and cognitive effects, which determine the efficiency of subsequent learning. These influences on subsequent learning are called indirect effects of retrieval practice. Although indirect effects have rarely been studied in isolation, there is already a large body of research from other research traditions that allows to draw conclusions about how retrieval practice affects subsequent learning. In this article, we compile and explain metacognitive, motivational, and learning goal-specific effects. We emphasize in particular the effects on meaningful learning that aims at understanding. In our overview, it gets clear that there is no single ideal way to exploit indirect effects of retrieval practice. Depending on the targeted learning goals and learner characteristics, different types of indirect retrieval practice are recommendable, as different types of retrieval have different strengths and weaknesses with respect to specific goals. Both teachers and learners should be aware of the specific mechanisms of the different indirect effects evoked by retrieval practice to deliberately optimize learning behavior.
\end{abstract}

Keywords Indirect testing effects - Metacognitive monitoring - Motivation · Learning goal-specific effects

\section{Einleitung}

Lehrkräfte sollten mehr Aufgaben, die den Abruf von kürzlich gelernten Inhalten erfordern, in ihren Unterricht einbauen. So ähnliche Sätze kann man in Blogbeiträgen oder wissenschaftlichen Arbeiten (z.B. Roediger und Butler 2011) zum sogenannten Testing Effekt finden. Der Testing Effekt beschreibt das Phänomen, dass Abrufübungen das Vergessen von neu gelernten Inhalten im Vergleich zu erneutem Lesen der Inhalte (restudy) mittelfristig verlangsamen kann. Wir können Inhalte demnach länger behalten, wenn wir diese Inhalte aus unserem Gedächtnis abgerufen haben. Dieses Phänomen tritt auch dann auf, wenn kein weiteres Lernen (z. B. um Wissenslücken zu schließen) nach dem Abrufen mehr möglich ist (Rowland 2014).

Der Testing Effekt hat in den letzten Jahren viel Aufmerksamkeit erfahren. Dabei wurde ein Bereich dieser Effekte häufig nicht explizit behandelt: die indirekten Effekte der Abrufübungen (Arnold und McDermott 2013). Dieser Begriff umfasst (vor allem) metakognitive und motivationale Effekte, die das Abrufen auf die Effizienz des darauffolgenden Lernens hat, also auf alle die Situationen, in denen ein erneutes Lernen nach dem Abruflernen möglich ist. Beispielsweise können Tests mit Abrufübungen den Lernenden aufzeigen, dass sie noch unbemerkte Wissenslücken haben. Dieses Aufzeigen reduziert die generelle Tendenz zur Überschätzung des eigenen Lernerfolgs und kann das folgende Lernen dadurch verbessern. Da in Schule und Hochschule Situationen, in denen erneutes Lernen nach dem Abruf erfolgt, sehr häufig vorkommen, möchte die vorliegende Arbeit diese Effekte in den Vordergrund stellen. Wir werden herausstellen, warum gerade bei bedeutungshaltigem 
Lernen, bei dem das Verstehen des Inhaltes im Fokus steht, die indirekten Effekte von besonderer Bedeutung sind. Wir werden beleuchten, welche Effekte erreicht werden können und wie diese erreicht werden können. Dazu werden Metakognition, Motivation, Lernzielkommunikation sowie Merkmale der Lernenden beleuchtet, die entscheidenden Einfluss auf den Lernerfolg haben können und beim Einsatz von Abrufübungen betrachtet werden sollten. Wir werden praktische Empfehlungen dazu abgeben, wie die Effekte einzeln sowie in ihrer Wechselwirkung so eingesetzt werden können, dass sie im Unterricht Anwendung finden können.

\section{Warum ist die Differenzierung direkter und indirekter Effekte gerade bei bedeutungshaltigem Lernen wichtig?}

Abrufeffekte wurden in unzähligen Studien untersucht und die Befunde in mehreren Metaanalysen dargelegt (Adesope et al. 2017; Pan und Rickard 2018; Rowland 2014; Yang et al. 2021). Obwohl indirekte Effekte der Abrufübungen ähnlich wie der direkte Testing Effekt bereits seit mehreren Jahrzehnten analysiert worden sind (z. B. Izawa 1966, 1970), sind im Hinblick auf indirekte Effekte noch vielen Fragen offen. Die in der Literatur zu findenden weniger konkreten Empfehlungen zu den indirekten Effekten sind unter anderem darauf zurückzuführen, dass die anfängliche Forschung zu Abrufübungen sehr grundlagenorientiert war. Dieser Umstand führte dazu, dass häufig recht artifizielle Bedingungen für die Untersuchung gewählt wurden, welche durch ihre spezielle Ausgestaltung die Effekte teilweise überschätzten. Bisweilen waren direkte und indirekte Effekte auch nicht voneinander differenzierbar. So wurde beispielsweise häufig das Erlernen von Wortlisten, bei dem sich mehrere Zyklen wiederholten Lernens und Abrufens abwechselten, auf einen verzögerten Erinnerungstest untersucht (z.B. Roediger und Karpicke 2006). Diese Methode war sehr erfolgreich, jedoch können die direkten und indirekten Effekte nur schwer getrennt bewertet werden. Solche Experimente können deshalb keine Einschätzung darüber gegeben, wie die indirekten und direkten Effekte jeweils das Lernen beeinflussen.

Auch wird die Bedeutung der indirekten Effekte für den Lernerfolg bisher teilweise als gering eingeschätzt. In vielen Arbeiten zum Thema Abrufübungen werden die indirekten Effekte lediglich als Feedbackeffekte interpretiert und nicht differenziert als Lerngelegenheit analysiert (z. B. Roediger und Butler 2011). Insbesondere bei den grundlagenorientierten Wortlistenexperimenten scheint man diese Effekte zu unterschätzen. In den meisten dieser Experimente wurden die richtigen Wortkombinationen als Feedback präsentiert. Dieses Feedback erfüllt in einem solchen Paradigma vermutlich eine deutlich bedeutendere Rolle, als nur die Lernenden zu informieren, ob ihre Antwort richtig oder falsch war. Sie kann als erneute Gelegenheit zum Lernen genutzt werden. Wie wichtig die erneute Darbietung des Materials ist, legen auch bereits veröffentlichte Metaanalysen nahe, die die Abrufübungen in unterschiedlichen Formen bewerten (z. B. Rowland 2014). Dabei zeigt sich, dass das Hinzufügen einer oder mehrerer erneuter Lerngelegenheiten die Effektstärke, die das Abrufen auf das Lernen hat, nahezu verdoppelt ( $g_{\text {gesamt }}=0,39$ auf $g$ mit Feedback $=0,73$; Rowland 2014). Eine weitere Metaanalyse, die ausschließlich Untersuchungen im Schulkontext einbezog, konnte diese Ergebnisse bestätigen. Es konnte eine ähnliche, 
wenn auch etwas geringere Verbesserung der Lernleistung festgestellt werden, wenn nach dem Abruf eine erneute Lerngelegenheit dargeboten wurde $\left(g_{\text {gesamt }}=0,37\right.$ auf $g_{\text {mit Feedback }}=0,57$; Yang et al. 2021).

In der Praxis mag die Differenzierung zwischen direkten und indirekten Effekten zunächst nicht allzu wichtig erscheinen. Als Lehrender mag man sich fragen: Wenn die Kombination aus direkten und indirekten Effekten gemeinsam erfolgreich evaluiert wurde, warum sollte man nicht direkt diese Kombination verwenden? Für Vokabellisten und einfache Fakten kann dies vielleicht auch in der Praxis so umgesetzt werden. Wenn es jedoch um bedeutungshaltiges Lernen geht, bei dem Lernende Verständnis erwerben sollen, sind die Inhalte häufig komplexer und die Lernmaterialien länger. Mit einem solchen Lernmaterial ist eine sich wiederholende Sequenz aus Lernen und Abrufen sehr aufwändig, da jede einzelne Phase mehr Zeit benötigt. Durch diesen erhöhten Zeitbedarf werden die Entscheidungen darüber wichtiger, wie sinnvoll eine erneute Lernphase ist, um die zur Verfügung stehende Zeit effizient zu nutzen. Außerdem bietet umfangreicheres Lernmaterial größere Freiheiten darin, die Abrufübungen und die erneute Lerngelegenheit zu gestalten, um günstig auf das darauffolgende Lernen einzuwirken. Während bei Vokabeln der Abruf und das erneute Lernen in den meisten Fällen lediglich in der Präsentation des zu erinnernden Wortes besteht, gibt es weitaus mehr Möglichkeiten bedeutungshaltig gelernte Inhalte abzurufen (z.B. unterschiedliche Fragentypen, Bearbeitung von Problemen ohne Zuhilfenahme des Buches, etc.) und auch unterschiedliche Wege, die Inhalte der erneuten Lerngelegenheit zu gestalten (z. B. Antwort auf gestellte Frage/Problem zur Verfügung stellen, Text erneut präsentieren, anderes Präsentationsmedium verwenden, etc.).

\section{Welche indirekten Effekte sind besonders relevant?}

Die indirekten Effekte werden mit einem ähnlichen Forschungsdesign wie die direkten Effekte untersucht. Dabei wird nach dem Abrufversuch eine erneute Lernmöglichkeit angeboten. Bisweilen wird das erneute Lernen auch nicht mehr realisiert, es werden aber Voraussetzungen dafür untersucht, eine potenzielle erneute Lerngelegenheit produktiv zu nutzen (z. B. das Bewusstsein für Wissenslücken oder Motivation; Endres et al. 2020; Little und McDaniel 2014; Tullis et al. 2013). Mit anderen Worten, in diesen Experimenten wurden lediglich Variablen erfasst, die sich auf die Nutzung einer erneuten Lerngelegenheit auswirken sollten, ohne dass die Lerngelegenheit tatsächlich stattgefunden hat. Auch in der Metakognitionsforschung werden solche Lernvoraussetzungen (z. B. Judments of Learning) vielfach fokussiert, ohne dass die Auswirkungen auf das darauffolgende Lernen analysiert werden. Dieses Vorgehen ermöglicht es zumindest, die direkten und indirekten Effekte des Abruftrainings innerhalb eines Experiments zu einem bestimmten Zeitpunkt im Lernprozess separat abzuschätzen. Die indirekten Effekte sind besonders bei bedeutungshaltigem Lernen von besonderem Interesse. Warum dies so ist, wird im Folgenden aus Sicht der Forschung zum Abruflernen und aus der Sicht des selbstregulierten Lernens dargestellt. 


\subsection{Relevanz aus Sicht der Forschung zum Abruflernen}

Das Einbinden von erneuten Lernmöglichkeiten nach Abrufübungen kann laut Metaanalysen die Lerneffekte über verschiedene Lernmaterialien hinweg erhöhen (Rowland 2014). Betrachtet man lediglich die Ergebnisse zum paarassoziativen Lernen, wie Vokabeln oder einfachen Fakten, so reicht es aus, lediglich die korrekte Antwort zu präsentieren, um einen Lerneffekt zu erzielen. Dies trifft nicht auf bedeutungshaltiges Lernen zu, wie eine Metaanalyse zu Abrufübungen beim bedeutungshaltigen Lernen zeigt (Pan und Rickard 2018). Wird nur die Antwort präsentiert, zeigt sich kein bedeutsamer Effekt. Um verständnisfördernde Effekte zu erzielen, ist es notwendig umfangreichere erneute Lerngelegenheiten anzubieten. Im Bereich des bedeutungshaltigen Lernens werden die erneute Präsentation des gesamten Lernmaterials oder zusätzliche Erklärungen zur gegebenen Antwort benötigt (vgl. das Konzept des Elaborative Feedback; Pan und Rickard 2018). Ein reines Bereitstellen der richtigen Antwort führt im Kontext dieses komplexeren Lernens sogar dazu, dass Lernende weniger lernen, als wenn sie kein Feedback bekommen (negative Effekte von Correct Answer Feedback; Pan und Rickard 2018).

Zudem sind die Freiräume, die Lernende beim erneuten Lernen haben, bei bedeutungshaltigem Lernen deutlich größer als beim Wortlistenlernen. Beispielsweise sind die Freiheiten beim erneuten Lernen aus einem Text deutlich größer als beim eindeutigen Lernen einer Vokabel. Deshalb sind die genutzten Strategien, die während des erneuten Lernens genutzt werden, unterschiedlich. Außerdem können beim bedeutungshaltigen Lernen verschiedene Lernende aus unterschiedlichen Gründen Wissenslücken aufweisen. So können bei einer Lernenden beispielsweise bereits zuvor falsch verstandene Konzepte zu späteren Verständnisproblemen und damit Wissenslücken führen, während eine andere Lernende lediglich mit dem Zusammenführen der Inhalte Probleme hat, die einzelnen Inhalte aber gut verstanden hat. Obwohl beide ggf. an derselben Stelle scheitern, benötigen sie unterschiedliche Informationen, um ihre Wissenslücke zu schließen. Deshalb sollte nach Verständnisfragen eine größere Bandbreite an Informationen während des erneuten Lernens bereitgestellt werden, d.h. es sind größere Ausschnitte der Inhalte notwendig, um substanzielle Effekte zu erzielen. Beim Vokabellernen mag hingegen jeweils die zugehörige Bedeutung als erneute Lerngelegenheit ausreichen.

Diese Ergebnisse und Interpretationen zeigen, dass die Entscheidung, wie erneutes Lernen bei bedeutungshaltigem Lernen zu gestalten ist, von mehreren Aspekten des Lernmaterials abhängig ist. Um mithilfe didaktischer Maßnahmen indirekte Effekte zu nutzen, können wir auf Forschung zu bekannten Mechanismen zurückgreifen, die zu indirekten Effekten beitragen. Dabei können wir nicht nur auf Untersuchungen zu indirekten Abrufübungen zurückgreifen. Auch aus anderen Bereichen der Lernforschung gibt es Erkenntnisse, die Aufschluss darüber geben, wie erneute Lerngelegenheiten beschaffen sein sollten. Diese werden im Folgenden genauer erläutert. 


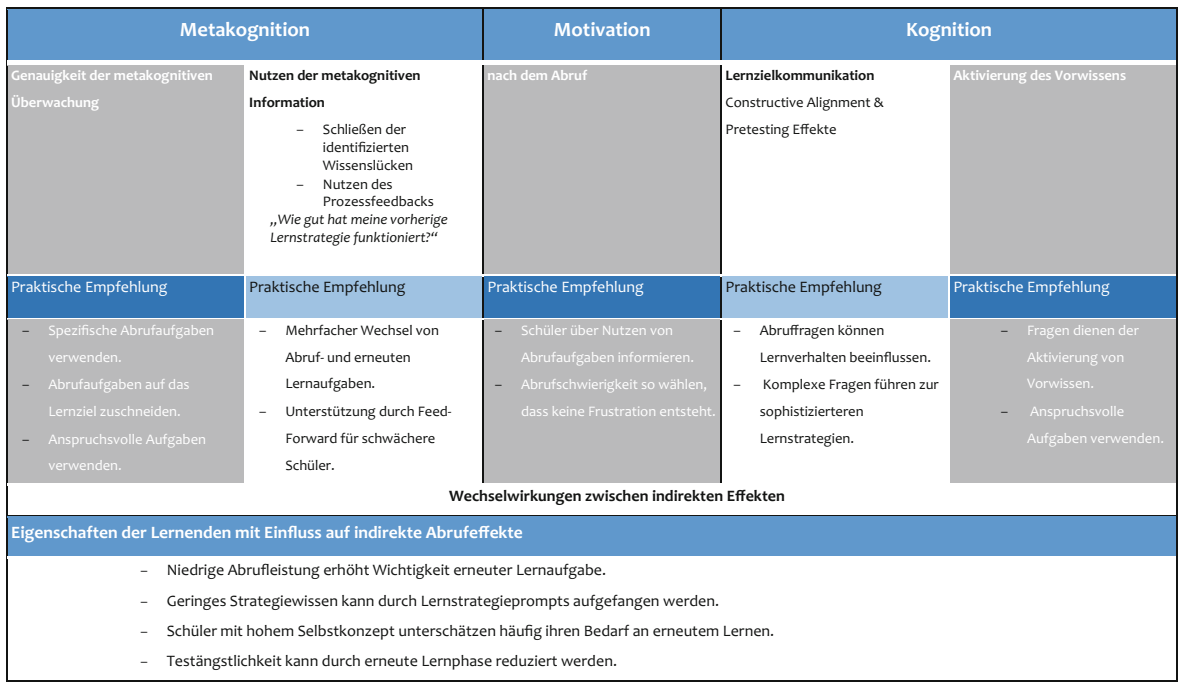

Abb. 1 Übersicht über indirekte Effekte und daraus abgeleitete Praktische Empfehlungen

\subsection{Relevanz aus Sicht des selbstregulierten Lernens}

Bei Abrufübungen aus bedeutungshaltig gelerntem Material sollte eine erneute Lerngelegenheit also mehr Informationen enthalten als für die reine Beantwortung der Frage notwendig ist (Endres und Renkl 2015). Wenn Informationen aus längeren Texten Inhalt der Abrufübungen sind, so werden im drauffolgenden Lernen Selbstregulationsprozesse benötigt, um die relevanten Aspekte des Textes nochmals zu lernen. Die Lernenden müssen entscheiden, welche Inhalte sie noch einmal vertiefen wollen und wie sie mit den Inhalten arbeiten. Diese Freiräume entstehen automatisch, wenn längere Texte oder Erklärungen verwendet werden, da es unterschiedliche Strategien gibt, um längere Texte zu verarbeiten. Insofern ist es sinnvoll, den Nutzen indirekter Abrufeffekte mit ähnlichen Konstrukten zu analysieren, wie sie beim selbstgesteuerten Lernen verwendet werden. Dazu zählen die metakognitive Überwachung, die Verwendung der metakognitiven Information, um Wissenslücken zu schließen (Regulation), und die Motivation der Lernenden (Winne und Hadwin 1998; Zimmerman 1990). Zusätzlich wird in den nächsten Abschnitten darauf eingegangen, wie die Eigenschaften der Lernenden und die Lernzielkommunikation das folgende Lernen beeinflusst (siehe Abb. 1).

\section{Genauigkeit der metakognitiven Überwachung}

Lernende haben häufig große Schwierigkeiten ihren aktuellen Lernstand akkurat einzuschätzen (de Bruin und van Merriënboer 2017). Dieser Prozess des Überwachens ist jedoch beim selbstgesteuerten Lernen von großer Relevanz: Nur wenn Lernende ihren aktuellen Lernstand kennen, können sie anschließend effizient und gezielt identifizierte Wissenslücken schließen (Winne und Hadwin 1998). Deshalb 
ist die Genauigkeit der metakognitiven Überwachung von besonderem Interesse, wenn indirekte Effekte der Abrufübungen bei bedeutungshaltig gelernten Materialien eigesetzt werden soll. Abrufübungen können einen Einfluss auf diese Genauigkeit haben. Nach dem Abrufen von Inhalten haben Lernende in der Regel eine bessere Vorstellung davon, wie gut sie das Thema gelernt haben und wie gut sie in einer künftigen Abrufaufgabe abschneiden werden (z. B. Hays et al. 2013; King et al. 1980; Lovelace 1984; Roediger und Karpicke 2006). Zudem stärken Abrufübungen das Vertrauen in die korrekt gegebenen Antworten und deren akkurate metakognitive Beurteilung (Barenberg und Dutke 2019; Shaughnessy und Zechmeister 1992).

Dabei ist es nicht zwingend notwendig, dass die Abrufübungen von einer Lehrkraft oder einem computerbasierten System bewertet wird. Das implizite Feedback während der Aufgabenbearbeitung kann ausreichen, um die metakognitive Überwachung zu verbessern. Mit implizitem Feedback ist das Feedback gemeint, welches die Lernenden automatisch während der Aufgabenbearbeitung über ihren Wissenstand gewinnen, ohne bewusst an die Überwachung zu denken oder zusätzliche Strategien zu nutzen. Bei Abrufübungen wird das Feedback zur Aufgabenleistung typischerweise indirekt über metakognitive Hinweisreize (metacognitive cues) abgeleitet. $\mathrm{Zu}$ diesen metakognitiven Hinweisreizen zählt insbesondere, wie flüssig es sich für die Lernenden anfühlt, die Inhalte aus dem Gedächtnis abzurufen (Koriat 2007).

Die Lehrenden können den Prozess des metakognitiven Überwachens zusätzlich beeinflussen, indem sie explizites Feedback geben. Wie dieses explizite Feedback die Metakognition der Lehrenden beeinflusst, wird im Rahmen der Forschung zu formativem Assessment untersucht. Formatives Assessment umfasst Methoden, die den aktuellen Lernstand der Lernenden abbilden; dabei wird besonderes Augenmerk auf die Diagnose der individuellen Leistung sowie auf ein darauf basierendes explizites Feedback gelegt. Implizites und explizites Feedback haben die gleiche Funktion. Beide Formen können die metakognitive Überwachung steigern und auBerdem Einfluss auf motivationale Faktoren nehmen (Moss und Brookhart 2019). Die Literatur zum formativen Assessment macht komplexe Vorhersagen darüber, wie Feedbackmerkmale das folgende Lernen beeinflussen (für einen Überblick siehe Narciss 2020). In diesem Artikel fokussieren wir zunächst das implizite Feedback.

Die metakognitiven Effekte von Abrufübungen sind vermutlich die Effekte, die den Lernenden am meisten bewusst sind. Verschiedene Studien konnten zeigen, dass Studierende Abrufaufgaben häufiger aufgrund ihrer metakognitiven Effekte verwenden (d.h. überprüfen, was man schon weiß) als aufgrund der direkten Effekte der Wissenskonsolidierung (Karpicke et al. 2009; Kuhbandner und Emmerdinger 2019; Rivers 2020; Roediger und Karpicke 2006; Tullis und Maddox 2020).

Weniger bekannt ist jedoch, dass auch die spezifische Art des Abrufens einen Einfluss auf die Genauigkeit der Überwachung hat. Wird eine unspezifische Abrufübungsaufgabe verwendet (z.B. eine freie Abrufaufgabe, an welche Textinhalte man sich noch erinnert), so ist das inhärente Feedback der Aufgabe relativ gering und Lernende erhalten weniger Rückmeldung über ihre vorhandenen Wissenslücken. Beispielsweise bemerken Lernende nicht, dass sie bei der Beantwortung einer unspezifischen Aufgabe einige wichtige Informationen ausgelassen haben, weil sie in der gegebenen Antwort über verschiedene andere Inhalte des Textes schreiben 
konnten. Der Prozess des Abrufens ist deshalb trotz vorhandener Wissenslücken flüssig. Infolgedessen bekommen die Lernenden den Eindruck, dass ihre Antworten gut waren, obwohl wichtige Teile des Wissens fehlten (Koriat 2012). Werden spezifischere Abrufübungsaufgaben verwendet (z. B. eine Kurzantwortaufgabe mit einem speziellen Inhalt), so ist das inhärente Feedback höher. Decken beispielsweise mehrere Kurzantwortaufgaben den gleichen Inhaltsbereich wie eine freie Abrufaufgabe $\mathrm{ab}$, erfordern die spezifischen Aufgaben detailliertere Antworten zu jedem Aspekt. So werden den Lernenden Wissenslücken eher bewusst. Außerdem ist der Erinnerungsprozess bei der Bearbeitung der spezifischen Aufgaben weniger flüssig. Das inhärente Feedback, das die Lernenden von spezifischen Aufgaben erhalten, ist also höher und gibt ihnen eine akkuratere Einschätzung über ihren Wissensstand (Endres et al. 2020). Je genauer die Fragen die wichtigen lernrelevanten Elemente abdecken, desto deutlicher zeigen sie den Lernenden noch vorhandene Wissenslücken auf, die zu schließen wären. Dies hilft den Lernenden, anschließend diese Wissenslücken gezielt zu schließen.

Es gibt es kaum Forschung, die diese metakognitive Funktion isoliert beleuchtet. In den meisten Fällen werden indirekte metakognitive Effekte mit direkten Effekten zusammen zur Interpretation der gefundenen Lerneffekte genutzt. Dabei könnte spezifisches Wissen darüber, wie Lernende selbständig mit Abrufaufgaben ihr Wissen überwachen können, hilfreiche Empfehlungen für Lehrende wie Lernende geben. Hier könnten besonders die Erkenntnisse aus Untersuchungen zum formativen Assessment genutzt werden, um unterschiedliche Arten der Abrufübungen und die Präsentation von erneuten Lerngelegenheiten so zu kombinieren, dass Lernende ein möglichst akkurates Bild ihres aktuellen Lernstandes haben. Im Rahmen des formativen Assessments ist es zentral eine systematische Erhebung des Leistungstandes durchzuführen (Souvignier und Hasselhorn 2018), dessen Systematik den Lernenden anschließend mitgeteilt wird, um größere Lerneffekte zu erzielen (Kingston und Nash 2011). Dieses explizite Deutlichmachen der Bewertungsstrukturen könnte auch in abrufbasierten Lerngelegenheiten den Lernenden die metakognitive Überwachung ihres Lernstandes erleichtern.

Praktische Empfehlungen Wie kann man diese Erkenntnisse aus der Forschung nun im Unterricht umsetzen? Die Überwachung des eigenen Lernstandes ist für Lernende eine Herausforderung, sie kann aber durch Abrufübungen erleichtert werden. Lernende wissen nach Abrufaufgaben besser, welche Inhalte sie schon beherrschen und welche Inhalte ihnen noch Schwierigkeiten machen. Wenn man Abrufübungen einsetzt, dann kann man die Überwachung zusätzlich verbessern, indem man sicherstellt, dass die Abrufaufgaben spezifisch nach den zentralen Lerninhalten fragen. Je spezifischer die Abrufaufgaben die zentralen Inhalte fokussieren, desto genauer ist die metakognitive Überwachung der Lernenden über ihren aktuellen Kenntnisstand zu diesen zentralen Inhalten. Mit anderen Worten, wenn im Unterricht also spezifische Inhalte besonders relevant sind, dann sollten genau diese Inhalte in Abrufübungen adressiert werden. Dabei ist es entscheidend, dass die Abrufaufgaben anspruchsvoll genug sind, um wichtige Wissenslücken aufdecken zu können. Eine zu einfache Aufgabe (z.B. Abruf unmittelbar nach der Wiederholung der Inhalte) ist nicht die optimale Basis für die metakognitive Einschätzung der Lernenden, 
vielmehr sollte der Abruf beispielsweise mit einer gewissen Verzögerung, die ihn etwas herausfordernder macht, stattfinden. Dabei ist es nicht unbedingt nötig den Lernenden explizites Feedback über die Richtigkeit ihrer abgerufenen Inhalte zu geben. Auch implizites Feedback während der Aufgabenbearbeitung kann helfen, mit Abrufaufgaben die Überwachung zu verbessern. Dies macht Abrufübungen besonders in selbstregulierten Lernkontexten besonders hilfreich, weil Lernende mit spezifischen, anspruchsvollen Aufgaben selbständig eine gute Einschätzung ihres Wissenstandes erhalten können. Möchten Lehrende die Überwachung weiter unterstützen, so können sie zusätzlich explizites Feedback geben. Hierbei sollten die Prinzipien des formativen Assessments beachtet werden und beispielsweise die Systematik der Bewertung kommuniziert werden. Dies kann besonders für Lernende mit geringem Lernstrategiewissen hilfreich sein (Athanases und Achinstein 2003).

\section{Verwendung der metakognitiven Information}

Abrufübungen können nicht nur relevante Wissenslücken aufzeigen, sondern auch zur Einschätzung des eigenen Lernprozesses (z. B. den zuvor genutzten Lernstrategien) und dessen Veränderung beitragen. In beiden Fällen haben Abrufübungen einen Einfluss darauf, wie Lernende ihr anschließendes Lernen gestalten. Aus Untersuchungen zu selbstreguliertem Lernen kann man jedoch annehmen, dass Lernende Schwierigkeiten haben die Informationen, die sie durch den Abruf bekommen, optimal für das erneute Lernen zu nutzen (z. B. Winne 2011).

\subsection{Nutzen von identifizierten Wissenslücken}

Wie bereits im vorherigen Abschnitt beleuchtet, erhöht der Abruf die Genauigkeit der metakognitiven Überwachung. Wenn Lernende unterscheiden können, welche einzelnen Inhalte sie wie gut beherrschen, nutzen sie ungeachtet des Ursprungs dieser metakognitiven Unterscheidung (z. B. durch implizites Feedback während einer Abrufaufgabe oder formatives Assessment) ähnliche Regulationsstrategien. Ist die Selbsteinschätzung des Lernstandes für einen spezifischen Inhalt sehr hoch, werden diese Inhalte nicht erneut gelernt. Für Inhalte, die noch nicht komplett verstanden sind, beginnen Lernende meist bei dem noch am besten verstandenen Inhalt arbeiten sich zum am wenigsten verstandenen Aspekt durch (Proximal Learning Model: Metcalfe und Kornell 2005).

Es konnte bereits gezeigt werden, dass das Schließen von Wissenslücken einen essenziellen Anteil der indirekten Lerneffekte von Abrufübungen darstellt (Endres et al. in prep). Werden nach einer Lernaufgabe unterschiedliche Abrufaufgaben verwendet, die sich in ihrer Sensitivität für Wissenslücken unterscheiden (z. B. spezifische und globale Aufgaben), dann kommen Lernende zu unterschiedlich akkuraten metakognitiven Überwachungsergebnissen. Nach spezifischeren Abrufaufgaben sind ihre Einschätzungen akkurater. Die Unterschiede in der Akkuratheit der metakognitiven Einschätzungen sind, wie in einer Mediationsanalyse in der genannten Studie gezeigt wurde (Endres et al. in prep), ausschlaggebend dafür, dass Lernende effizient 
aus einer darauffolgenden Lernphase lernen und einen höheren Lernerfolg erzielen. Dabei werden besonders die zuvor nicht erinnerten Inhalte besser wiedergegeben.

\subsection{Nutzen des Prozessfeedbacks}

Auch der vorherige Lernprozess kann durch Abrufübungen evaluiert werden. Forschung zum sogenannten Forward Effect of Testing (auch Test Potentiated New Learning) konnte zeigen, dass Testen auch einen höheren Lernerfolg in Bezug auf neue, noch zu erlernende Inhalte bewirken kann (für Überblicksarbeiten siehe Chan et al. 2018; Pastötter und Bäuml 2014; Yang et al. 2018). Dieser Forschungsbereich untersucht also den Einfluss, den Abrufübungen zu einem spezifischen Inhalt auf darauffolgende Lernprozesse bei anderen Inhalten hat. Im Gegensatz zu Transfereffekten, die sich auf die Anwendung erlernten Wissens in nachfolgenden, aber komplexeren Anwendungssituation beziehen (z. B. Problemlösung), fokussiert der Forward Effect of Testing den metakognitiven Aspekt der Evaluation des vorherigen Lernprozesses für zukünftiges Lernen. Es geht also bei Studien zum Forward Effect of Testing nicht darum Wissen in einer anderen Situation anzuwenden (Transfereffekte) sondern darum die Strategie, die zum Lernen verwendet wurde, zu evaluieren, und gegebenenfalls anzupassen. Eine exemplarische Studie aus diesem Bereich lässt zwei Gruppen zuerst Text A zum Thema Steuerpolitik lernen. Anschließend werden in einer der Gruppen Abruffragen zum Thema Steuerpolitik gestellt. Danach folgt Text B zum Thema Arbeitsrecht, der von beiden Gruppen gelesen wird. Abschließend erfolgt in beiden Gruppen der Lerntest zum Thema Arbeitsrecht. Der Forward Effect of Testing beschreibt das Ergebnismuster, dass die getestete Gruppe die Gruppe ohne Test in ihrem Lernerfolg zum Thema Arbeitsrecht übertrifft. Dieser Effekt konnte mit unterschiedlichen Materialien belegt werden (Lerntexte: Healy et al. 2017; Wissman et al. 2011; Vorlesungsaufzeichnungen: Jing et al. 2016; Szpunar et al. 2013; Yue et al. 2015).

Besonders bei bedeutungshaltigen Lernmaterialien wird der Lerneffekt darauf zurückgeführt, dass Lernende ihre Lernstrategie, die sie beim Lernen des ersten Textes angewandt haben, beim Lernen des zweiten Textes anpassen (Wissman et al. 2011). Wenn Lernende also den Text zum Thema Steuerpolitik lesen und anschließend dieses Thema abrufen müssen, merken sie, ob ihre bisherige Lernstrategie für diese Art des Lernmaterials erfolgreich war. In der nun folgenden neuen Lernphase zum Thema Arbeitsrecht wenden sie die potenziell angepasste Lernstrategie an und erzielen so einen besseren Lerneffekt. Gerade beim Scheitern der Abrufaufgaben zeigt sich, dass Lernende ihre Lernstrategie in einer darauffolgenden Lerngelegenheit zu einem anderen Thema anpassen und verbessern (Wissman et al. 2011).

In dieser Forschungstradition wurden Regulationseffekte lediglich auf neues Lernen untersucht, um möglichst unabhängig von direkten Abrufeffekten den Lernerfolg bewerten zu können. Für die Praxis ist es aber natürlich auch interessant, wie indirekte und direkte Effekte hier zusammenwirken. Es ist sehr wahrscheinlich, dass ähnliche Effekte auf den Lernprozess auch für bereits abgerufene Inhalte auftreten. Diese Annahme müsste jedoch in weiterer Forschung direkt getestet werden. Dafür wäre ein kreativer Weg nötig, um zwischen den Effekten eines veränderten Lernprozesses und anderen Effekten zu unterscheiden. Beispielsweise könnten kognitive 
Veränderungen durch den Abruf oder die erhöhte metakognitive Überwachung und das Schließen der Wissenslücken den Lerneffekt mit beeinflussen und somit den Rückschluss auf den Regulationseffekt erschweren. Hier könnten beispielsweise eine Laut-Denken Methodik helfen, bei der die Lernenden ihre Bearbeitung während des erneuten Lernens ihre Gedanken laut aussprechen. So könnte man möglicherweise den spezifischen Effekten der Strategieanpassung nachgehen. Ebenso wäre die Methode des cued retrospective reporting denkbar. In dieser Methode werden zunächst die Blickbewegungen der Lernenden während des erneuten Lernens aufgenommen. In einer anschließenden Phase wird den Lernenden die Aufzeichnung der Blickbewegungen anschaulich präsentiert und sie können ihren Lernprozess nun kommentieren. Der Vorteil dieser Erhebungsmethode gegenüber dem lauten Denken ist, dass sie die Lernprozesse selbst nicht beeinflusst (z. B. Bender et al. 2021).

Des Weiteren können Abrufübungen typische Verzerrungen verringern, denen Lernende häufig unterliegen. Für gewöhnlich haben Lernende die Tendenz, in multimedialen Lernumgebungen, die sowohl Text als auch Bilder beinhalten, die Bilder zu vernachlässigen, selbst wenn diese hilfreich für das Lernen wären. Dieser Effekt konnte reduziert werden, indem man Lernende innerhalb einer solchen multimedialen Lernumgebung Abruffragen präsentierte (Eitel 2016). Ähnlich wie in einem Experiment zum Forward Effect of Testing konnten Lernende durch Abrufaufgaben ihren Prozess besser überwachen und haben beim Lernen nach Abrufübungen Bilder mehr in ihren Lernprozess einbezogen. Eine höhere Betrachtungszeit des Bildes war dabei ausschlaggebend für den Lernerfolg im zweiten Lerngebiet (Eitel 2016). Die Lernenden haben also auch hier ihren Lernprozess nach Abrufübungen angepasst.

Eine Metaanalyse zu Abrufübungen in bedeutungshaltigen Lernkontexten liefert außerdem Hinweise auf besonders förderliche Effekte, wenn Abrufübungen in mehreren konsekutiven Phasen z verschiedenen Themen implementiert wird. Wenn Abrufübungen in mehreren Fächern angewendet wurde, konnte ein größerer Effekt gefunden werden, als wenn es lediglich in einem Fach durchgeführt wurde ( $\mathrm{g}_{\text {ein Fach }}=0,389, \mathrm{~g}_{\text {mehrere Fächer }}=0,541$; Yang et al. 2021).

Die bereits erwähnte Forschung zum formativen Assessment macht deutlich, dass der Regulationsprozess der Anpassung der Lernstrategien weiter unterstützt werden sollte, um bestmöglich von der verbesserten metakognitiven Überwachung zu profitieren. So wäre es möglich, die Lernenden in Form eines Feed-Forward explizit darauf hinzuweisen, welche Teile des Textes sie noch nicht so gut verstanden haben oder in welcher Art und Weise sie bei Texten dieser Art lernen könnten. Dieses Feed-Forward sollte direkt in Zusammenhang mit ihrer Leistung in der Abrufübungsaufgabe gebracht werden (Kingston und Nash 2011). Beispielsweise sollte die Empfehlung einer metakognitiven Überprüfungsstrategie mit einem konkreten Fehler in der Abrufaufgabe in Verbindung gebracht werden. Dabei scheinen besonders schwächere Lernende von solchem Feed-Forward zu profitieren (Gezer et al. 2021). Im Besonderen scheinen leistungsschwache Lernende mehr von direkten und expliziten Rückmeldungen zu profitieren, da sie beim Lernen mehr Anleitung und Struktur brauchen (Shute 2008). Studien konnten häufiger größere Effekte solcher Interventionen bei schwachen Lernenden finden (Koedinger et al. 2010; Wang 2014). 
Praktische Empfehlungen Um für die Regulation sowohl Hinweise zum konkreten Lernstand als auch zum Lernprozess der Lernenden nutzbar zu machen, sollten Abrufübungen in mehreren konsekutiven Phasen implementiert werden, d.h. Phasen des Lernens und Abrufens sollten sich abwechseln. Nach einer Abrufaufgabe sollte also nicht nur das Material erneut verfügbar sein, sondern sogar weitere Abrufaufgaben nach dem erneuten Lernen eingebaut werden. Durch den Wechsel zwischen den Prozessen können die Lernenden sowohl die metakognitiven Informationen zu ihrem Wissen und wie auch zu ihren Lernstrategien nutzen. Die Abrufaufgaben sollten für eine möglichst optimale Regulation analog zum Überwachen des aktuellen Lernstandes anspruchsvoll und lernzielorientiert sein, damit die Lernenden die notwendigen Hinweise erhalten, um sich angemessen regulieren zu können. Zusätzliche Hinweise durch die Lehrenden in Form eines Feed-Forward können die Regulationsprozesse aller Lernenden unterstützen. Dies scheint besonders bei schwächeren Lernenden empfehlenswert.

\section{Motivation nach dem Abruf}

Ein weiterer wichtiger indirekter Effekt ist die veränderte Motivation nach der Abrufübung. Die Implementierung von Abrufübungen insbesondere in Universitätskursen kann Studierende dazu motivieren, regelmäßiger zu lernen (Roediger 2011). Während in vielen Kursen Studierende vor allem kurz vor den Klausuren versuchen, in massierter Form den gesamten Stoff zu erlernen (Blasiman et al. 2017), führte eine konstante Implementierung von Abrufübungen dazu, dass Studierende ein konstanteres Lernverhalten zeigen. Studierende halten dieses konstante Lernverhalten überraschenderweise auch dann aufrecht, wenn die Abrufübungstests nicht benotet werden, und sie sind motivierter zu lernen (Leeming 2002; Lyle und Crawford 2011).

Regelmäßiges Abruftraining scheint sogar ein positives Gefühl gegenüber der Lernaktivität des Abruftrainings hervorzurufen (McDaniel und Little 2019). Diese motivationalen Vorteile sind sehr überraschend, da Prüfungen, zu denen die implementierten Abrufübungen große Ähnlichkeit haben, normalerweise negativ konnotiert sind). Wenn die Lehrkraft jedoch deutlich macht, dass diese Abrufaufgaben den Zweck verfolgen, die Lernergebnisse zu verbessern, scheinen die Lernenden Abrufaufgaben wertzuschätzen und sie können zwischen summativen Tests und Abrufübungen unterscheiden.

Hier müssen zwei Erklärungsperspektiven zu diesen motivationalen Effekten verbunden werden. Die erste Perspektive bezieht sich auf Theorien zur Neugier (z. B. Wissensdeprivationshypothese; Rotgans und Schmidt 2011; Schmidt und Rotgans 2021). Diese Theorien nehmen an, dass ein Abruf möglichst nicht zu $100 \%$ erfolgreich sein sollte, um so die Neugierde der Lernenden für das Lerngebiet zu wecken und die Motivation, hier speziell das situationale Interesse, sich mit diesem Gebiet auseinanderzusetzen, zu steigern. Auch in diesem Bereich können Hinweise aus Metaanalysen zum Forward Effect of Testing herangezogen werden. In diesen Studien wurde ein Teil des Lerneffektes darauf zurückgeführt, dass das Erleben von Abruffehlern in früheren Tests ein metakognitives Bewusstsein für die Schwierig- 
keit des Lernmaterials allgemein auslöst. Das Bewusstsein für die Schwierigkeit den Abruf erfolgreich zu gestalten, motiviert die Lernenden dazu, mit mehr Engagement zu lernen (Cho et al. 2017; Yang et al. 2018). Diese Perspektive erklärt also, warum Abrufaufgaben, die Wissenslücken aufzeigen, motivierender sein können als ein vollständig erfolgreicher Abruf bzw. kein Abruf, weil diese beiden keine Abruffehler aufzeigen können.

Die zweite Perspektive bezieht sich auf den Unterschied zwischen unterschiedlichen Abrufaufgaben, die in diesem Fenster liegen (kein vollständiger erfolgreicher Abruf - kein Abruf). Diese Perspektive nimmt an, dass ein erfolgreicher Abruf als Erfolgserlebnis wichtig ist und die Selbstwirksamkeit der Lernenden erhöht. Selbstwirksamkeit resultiert vor allem aus unseren früheren Wahrnehmungen beim Ausführen einer ähnlichen Tätigkeit (Bandura 1997). Wenn Lernende Erfolgserlebnisse haben, erwarten sie auch, dass sie in der Zukunft bei ähnlichen Aufgaben erfolgreich sein werden (Schunk 1985). Hier konnte eine Untersuchung zeigen, dass im Sinne der zweiten Erklärungsperspektive ein erfolgreicher Abruf zu einer höheren Selbstwirksamkeit und zu höherem situativem Interesse führte (Endres et al. 2020).

Diese Erklärung der Motivation führt, in Verbindung mit den Befunden zur Metakognition, zu einem Dilemma: Wenn man sowohl Motivation als auch eine exaktere metakognitive Überwachung erreichen möchte, ergeben sich gegensätzliche Handlungsempfehlungen. Abrufübungen, die zu einer akkuraten metakognitiven Überwachung führen, sind häufig schwierig und geben den Lernenden nur geringe Erfolgserwartung. Ein Erreichen sowohl einer hohen Motivation als auch einer akkuraten metakognitiven Überwachung scheint daher innerhalb einer einzelnen Aufgabe schwer möglich (siehe dazu Endres et al. 2020).

Praktische Empfehlungen Insgesamt kann das Implementieren von Abrufübungen die Motivation von Lernenden steigern. Lehrende sollten also klar kommunizieren, welchen Zweck Abrufübungen auch bei komplexen Materialien haben können und wie sie Wissen und Metakognition verbessern. Wenn diese kommuniziert wird, bewerten Lernende summative Tests, wie Schulaufgaben und Klausuren, und Abrufübungsaufgaben bzw. formative Assessmentaufgaben unterschiedlich. Durch diese Kommunikation fühlen sich Lernende vor allem durch Abrufübungen motiviert. Im Unterschied zur metakognitiven Funktion sollte um die Motivation zu steigern die Aufgabenschwierigkeit der Abrufaufgaben sehr vorsichtig gewählt werden. Es gilt hier ein sensibles Gleichgewicht zu erreichen: Wissen die Lernenden schon die Antwort auf die meisten Aufgaben, wird keine zusätzliche Motivation erzeugt; wissen die Lernenden aber zu wenig, so reduziert sich ihre Motivation.

\section{Lernzielkommunikation: Constructive Alignment und Pretesting- Effekte}

Eine weitere Möglichkeit, mithilfe von Abrufübungen indirekte Effekte zu erzielen, ist die Vermittlung der Lernziele. Die Art der genutzten Abrufaufgaben vermittelt Lernenden indirekt, welche Ziele ein Lehrender verfolgt. Arbeiten zum Constructive Alignment (Biggs 1996) betonen, wie wichtig es ist, dass Lernziele und Ler- 
nassessment kompatibel sind, um das Lernverhalten von Lernenden zu beeinflussen (Leber et al. 2018). Dabei wird häufig die Konzeption von summativen Assessments wie Klausuren und Schulaufgaben erforscht. Aber auch Abrufaufgaben können Teil dieser indirekten Kommunikation zwischen Lehrenden und Lernenden sein. Das Bewusstsein für die Abhängigkeit von Abrufaufgaben und dem daraus folgendem Lernverhalten könnte genutzt werden, um indirekte Effekte von Abrufübungen besser zu verstehen. Nutzen Lehrende für die Abrufübungen eher faktenorientierte Fragen, dann werden Lernende in einer folgenden erneuten Lernsituation besonderen Fokus auf diese Aspekte legen. Konzentrieren sich Lehrende mit den Abrufübungen jedoch auf Verständnis oder Transfer, so werden komplexere Lernstrategien angewandt. Abruffragen könnten so die Lernenden in einer erneuten Lernphase zur Anwendung bestimmter Lernstrategien motivieren (vgl. Leber et al. 2018).

In diesem Zusammenhang weist die Literatur zu Pretestingeffekten auf relevante Befunde hin. In diesen Untersuchungen werden Testfragen zum folgenden Thema vor dem eigentlichen Lernen gestellt, um Lernenden den Fokus der darauffolgenden Lernaufgabe deutlich zu machen. Auf diese Art und Weise können inhaltsleitende Effekte von Abruffragen analysiert werden, ohne dass direkte Effekte des Abrufens großen Einfluss nehmen. In diesen Studien zeigt sich bei faktenorientierten Fragen für gewöhnlich lediglich ein Effekt auf die zuvor getesteten Inhalte (Bull und Dizney 1973; Hausman und Rhodes 2018; James und Storm 2019; McCrudden et al. 2005; Memory 1983; Pressley et al. 1990; Rickards 1976; Rothkopf und Billington 1979). Wenn hingegen komplexere, verständnisorientierte Fragen genutzt werden, erinnern Lernende auch die Inhalte häufiger, die nicht in den Pretestingfragen adressiert wurden, und der Lernerfolg insgesamt erhöht sich. Dies gilt aber nur dann, wenn der Fragentyp mit dem Fragentyp des Assessments übereinstimmt (St. Hilaire et al. 2019). Die positiven Effekte des Pretesting werden dabei auf eine tiefere Auseinandersetzung mit den Lerntexten zurückgeführt (Pan und Sana 2021).

Praktische Empfehlungen Abrufaufgaben sind Teil der Kommunikation von Lernzielen. Durch die Art der genutzten Abruffragen kann das Lernverhalten der Lernenden beeinflusst werden. Faktenorientierte Fragen führen zum Erlernen spezifischer Inhalte mit eher oberflächlichen Lernstrategien. Komplexe verständnisorientierte Fragen führen zur Anwendung sophistizierterer Lernstrategien. Welche Abrufaufgaben genau genutzt werden sollten, hängt vom Lernziel ab. Im Unterricht kann so das Lernverhalten durch Abrufaufgaben zusätzlich beeinflusst werden.

\section{Aktivierung des Vorwissens}

Eine weitere Erklärung für positive indirekte Lerneffekte ist, dass der Abruf von Wissen, welches gerade gelernt wurde, zu einer Aktivierung des bereits vorhandenen Vorwissens führen kann. Eine Aktivierung von Vorwissen führt häufig zu einer Verbesserung des nachfolgenden Lernprozesses. In Studien zur Vorwissensaktivierung wird das Lernen eines Textes mit vorheriger Aktivierung von relevantem Wissen und ohne eine solche Aktivierung untersucht. Es zeigt sich, dass wenn unmittelbar nach dem Lernen das Verständnis überprüft wird, der Lernerfolg größer ist wenn zuvor 
eine Aktivierung stattgefunden hat (Chi et al. 1994). Hier wird der Unterschied zu Studien deutlich, die direkte Abrufaufgaben untersuchen. In diesen Studien sind die Lerneffekte meist erst nach einigen Tagen oder Wochen Verzögerung beobachtbar (Rowland 2014). Verschiedene theoretische Modelle betonen die Wichtigkeit des Vorwissens im Lernprozess (Mayer 1997; Kintsch 1988; Graesser et al. 1994; Ausubel 1968). Wenn Lernende ihr Vorwissen aktivieren, dient dieses aktivierte Wissen als Rahmen für die Herstellung von Beziehungen zwischen dem Wissen, das sie bereits besitzen, und den neuen Informationen, die ihnen vermittelt werden. Dies erleichtert das Anknüpfen an bereits vorhandenes Wissen und der Wissenserwerb ist in der Folge effizienter (Renkl 2015). Das Phänomen, dass Vorwissen von Lernenden nicht aktiviert wird, ist vor allem bei Kindern gut erforscht. Kinder scheinen häufiger Defizite bei der strategischen Nutzung ihres Vorwissens aufzuweisen und profitieren deshalb besonders von wissensaktivierenden Maßnahmen (Barnes et al. 1996).

Der Abruf von Wissensinhalten aktiviert dabei nicht nur das Vorwissen und führt zu einem geringeren Vergessen (Carpenter et al. 2018). Abrufübungen verbessern in einigen Fällen auch das Behalten von Material, das nicht direkt abgerufen wurde. Wissen, das eng mit den abgerufenen Inhalten verbunden ist, wird ebenfalls besser erinnert. Der positive Effekt auf verbundenes Wissen, welches jedoch nicht direkt abgerufen wurde, wird als abrufinduzierte Erleichterung bezeichnet (Chan 2009). Wenn wir nach Inhalten in unserem Gedächtnis suchen, können wir mehrere Inhalte aktivieren, die mit dem Abrufziel verbunden sind (Carpenter 2009). Dieses Phänomen wird auch als Aktivierungsausbreitung (spreading activation) bezeichnet (Collins und Loftus 1975). Der Abruf von Inhalten führt zu einer besseren Verbindung zu verwandten Konzepten. Dieser Effekt ist jedoch in Studien nicht stabil (Pan und Rickard 2018). Das Behalten von nicht abgerufenen Inhalten scheint eng mit der Struktur des Lernmaterials verbunden zu sein. Bei vernetztem Lernmaterial, wie z.B. Texten, wird in der Regel eine abrufinduzierte Erleichterung festgestellt. Bei weniger vernetztem Material, wie z. B. unzusammenhängenden Listen oder Vokabeln als Lernmaterial, scheint dieser Effekt zu verschwinden und sich für das unmittelbare Lernen sogar umzukehren (Chan 2009).

Dabei stehen Aktivierungsausbreitung und die mentale Anstrengung, die für den Abruf investiert wird, in einer engen Verbindung. Ein höheres Ausmaß an investierter Anstrengung führt zu einer breiteren Aktivierungsausbreitung (Carpenter 2009) und damit auch zur Aktivierung von mehr Vorwissen. Neben den positiven Effekten auf das Vergessen (Endres und Renkl 2015) hat eine höhere Anstrengung also auch einen unmittelbaren Nutzen.

Praktische Empfehlungen Abrufaufgaben aktivieren Vorwissen für die darauffolgende Lernsituation. Dabei wird besonders bei zusammenhängendem Lernmaterial, wie es hier im Vordergrund steht, auch Vorwissen aktiviert, das mit dem abgerufenen Inhalt in Verbindung steht. Je anspruchsvoller der Abruf ist, im Sinne des Auslösens vermehrter mentaler Anstrengung, desto größer ist der Bereich des aktivierten Vorwissens. Dies gilt vor allem bei verständnisorientiertem und zusammenhängendem Wissen, wie es in diesem Themenheft im Fokus steht. Lehrende, die besonderes Augenmerk auf die Vorwissensaktivierung legen wollen, sollten anspruchsvolle Ab- 
rufaufgaben verwenden, die viele Suchprozesse auslösen, um so möglichst breites Vorwissen zu aktivieren. Dazu können beispielsweise Fragen dienen, bei denen mehrere Aspekte gleichzeitig berücksichtigt werden müssen.

\section{Wechselwirkungen zwischen den Effekten}

Der Forschungsstand erlaubt Aussagen über einzelne indirekte Effekte von bestimmten Abrufübungen. Bei einer integrierten Betrachtung indirekter Effekte können sich jedoch Zielkonflikte ergeben. Wie bereits dargelegt erhöhen beispielsweise spezifische Abrufaufgaben die metakognitive Überwachung, verringern aber gleichzeitig die Motivation im Sinne der Selbstwirksamkeit der Lernenden. Ein weiteres Beispiel ist, dass bei breiteren Abrufaufgaben Lernende schlechter konkrete Wissenslücken identifizieren können, gleichzeitig regen diese Fragen aber zu einer tieferen Auseinandersetzung mit der folgenden Lernphase an. Diese Wechselwirkungen machen deutlich, dass es nicht die eine Art von Abrufaufgaben gibt, die immer eingesetzt werden sollte. Je nach Lernziel und Eigenschaften der Lernenden sind unterschiedliche Arten von Abrufübungen zu empfehlen, um optimale Lernergebnisse zu erzielen. Sowohl Lehrende wie auch Lernende sollten um Effektmuster wissen, um ihr Lehrbzw. Lernverhalten ideal gestalten zu können.

Um die direkten Effekte der Abrufübung, die in den anderen Artikeln dieses Themenheftes besprochen wurden (Barenberg und Dutke; Roelle und Nückles; Rummer und Schweppe; in diesem Themenheft), optimal auszunutzen, sind teilweise entgegengesetzte Entscheidungen zu denen für die indirekten Effekte naheliegend. So erhöht ein hoher Abruferfolg häufig die Größe des direkten Testing Effekts, während anspruchsvolle Aufgaben die indirekten Effekte begünstigen. In Situationen, in denen eine erneute Lernsituation wahrscheinlich ist, ist deshalb ein Abruferfolg, der nahezu vollständig ist, nicht ideal, um beide Effekte nutzen zu können. Es zeigt sich, dass bereits ein Abruferfolg von rund dreiviertel des zu lernenden Inhaltes (Rowland 2014) für substanzielle direkte Abrufübungseffekte ausreicht. Es scheint daher sinnvoll diese Schwelle als Richtlinie für die Ausnutzung beider Effekte anzustreben. Für eine Nutzung der indirekten Effekte alleine ist diese Schwelle jedoch nicht nötig.

Um verschiedene Lernziele innerhalb einer Phase von Abrufübungen zu erreichen, kann es auch sinnvoll sein, eine Kombination aus verschiedenen Abrufaufgaben einzusetzen. Während in der Forschung zur genauen Bestimmung der ablaufenden Prozesse eine spezifische Art des Abrufes eingesetzt wird, so ist dies in der konkreten Anwendung wohl nicht immer sinnvoll. So wäre es zum Beispiel denkbar, dass mit spezifischen Fragen alle zentralen Wissenselemente abgedeckt werden, gleichzeitig einzelne dieser Aspekte aber mit komplexeren Fragen abgerufen werden müssen (siehe auch Lindner et al. 2015). So können Lehrende sicherstellen, dass die zentralen Konzepte gestärkt werden und gleichzeitig komplexe Lernstrategien angewendet werden. Weitere Forschung könnte diese Art von Kombination untersuchen, um noch konkretere Empfehlungen zu ermöglichen, wie Abrufübungen in den Unterricht eingebunden werden können. 


\section{Eigenschaften der Lernenden mit Einfluss auf indirekte Abrufeffekte}

Einige indirekte Effekte sind abhängig von individuellen Eigenschaften der Lernenden. Da der Abruferfolg bei Abrufübungen eine wichtige Rolle bei den indirekten Effekten spielt, ist das Vorwissen der Lernenden eine zu beachtende Größe. Lernende mit viel Vorwissen lernen bei sonst gleichen Lernphasen meist mehr aus einer ersten Lernphase (McNamara und Kintsch 1996). Deshalb werden bei derselben Abrufaufgabe Lernende mit viel Vorwissen meist auch einen höheren Abruferfolg haben als Lernende mit niedrigem Vorwissen (Spitzer 1939). In der Folge werden Lernende mit niedrigem Vorwissen mehr Wissenslücken identifizieren und eine geringere Motivation im Sinne von Selbstwirksamkeit aufweisen. Dem bereichsspezifischen Vorwissen kommt deshalb beim Abruflernen eine ganz besondere Bedeutung zu, weil gerade bei großen Unterschieden zwischen Lernenden teilweise entgegengesetzte Effekte auftreten oder einzelne Lernende von Abruf oder erneuten Lernphasen nicht profitieren können.

Neben bereichsspezifischem Wissen können aber auch übergreifende Fähigkeiten die indirekten Effekte von Abrufübungen beeinflussen. So scheinen Abrufübungen mit anschließender erneuter Lerngelegenheit vor allem für schwache Lernende von Vorteil zu sein (z. B. geringere Arbeitsgedächtniskapazität, Agarwal et al. 2017). In diesem Zusammenhang werden unterschiedliche Aspekte diskutiert, die der Grund dafür sein könnten, dass eine erneute Lernphase gerade für leistungsschwächere Lernenden nützlich ist. Der erste Aspekt ist, dass der ursprüngliche Abruferfolg nicht ausreicht, um einen direkten Abrufübungseffekt zu erzeugen (geringerer Testing Effekt bei niedrigem Abruferfolg, z. B. van Gog und Sweller 2015). Wenn Lernende nicht genug von direktem Abruf profitieren, so ist es sinnvoll, sie zusätzlich zu unterstützen. Dabei kann der geringe Abruferfolg durch geringere kognitive Fähigkeiten bedingt sein, die die schwachen Lernenden nicht befähigen, ausreichend aus der ersten Lernphase zu lernen (Renkl und Stern 1994). Dies kann an allgemeinen (teils angeborenen) Fähigkeiten liegen oder auch durch die kognitive Entwicklung in der Lebensspanne bedingt sein. Im Alter scheinen Lernende weniger von direkten Abrufübungen zu profieren, weil die Abrufaufgaben für sie sehr schwierig sind und nur ein geringer Abruferfolg erzielt wird (Vergleich bis 30- und über 50-Jährige; Guran et al. 2019). Auch bei sehr jungen Kindern, ist der Abruf häufig zu gering, um substanzielle Effekte zu erzielen (Vergleich von 8- und 12-Jährigen, Lechuga et al. 2006). Deshalb scheinen Lernende, die geringere kognitive Leistungsfähigkeit besitzen, besonders von indirekten Effekten zu profitieren.

Aber auch allgemeines Wissen über Lernstrategien ist relevant. Lernende weisen große Unterschiede in ihrer Erfahrung und ihrem Wissen über Lernstrategien für selbstreguliertes Lernen auf (Pintrich 2002). Wenn ein Lernender mit selbstreguliertem Lernen sehr erfahren ist, so lernt dieser, aufgrund der effizienteren Lernstrategien, bereits besser aus der ersten Lerngelegenheit vor dem Abruf. Dieser Lernerfolg befähigt die Lernenden mit gutem Strategiewissen einen höheren Abruferfolg in der Abrufübungsaufgabe zu erzielen. Diese Lernenden profitieren demnach weniger von einer zusätzlichen Lernphase nach dem Abruf. Zum anderen haben diese Lernenden eine bessere metakognitive Überwachungsfertigkeit. Sie sollten demnach 
weniger vom zusätzlichem (impliziten) Feedback durch Abrufübungen profitieren. Dem gegenüber brauchen Lernende mit schlechten metakognitiven Fertigkeiten die zusätzlichen metakognitiven Informationen, um sich gut überwachen zu können.

Es gibt jedoch auch Vorteile für Lernende mit hohem Lernstrategiewissen. Lernende mit niedrigem Strategiewissen scheitern häufig daran, adäquate Regulationshandlungen zu ergreifen, selbst wenn sie ein akkurates Bild ihres aktuellen Lernstandes haben. Daher können Lernende mit viel Lernstrategiewissen besser von der durch Abrufübungen unterstützten Metakognition profitieren. Insofern mag es sinnvoll sein, Lernende, die mit der Regulation Schwierigkeiten haben, dabei zu unterstützen. Hierzu könnten zum Beispiel Prompts verwendet werden, die die Regulationsstrategieanwendung anregen (z. B. Nückles et al. 2020; Müller und Seufert 2018). Außerdem könnten adaptive Lernsysteme genutzt werden, um individuelle erneute Lerngelegenheiten an den Regulationsbedarf der Lernenden anzupassen und im Sinne adaptiven Unterrichts unterschiedliche Materialien anzubieten (Kingston und Nash 2011; Renkl et al. 2015).

Vor allem wenn Abrufübungen nicht von einer Lehrkraft implementiert werden, haben Lernende mit einem hohen Lernstrategiewissen einen Vorteil. Diese Lernenden haben ein besseres konditionales Wissen darüber, wann Abrufaufgaben sinnvoll sind und wann diese einzusetzen sind. Wenn das Wissen nicht vorhanden ist, wann und wie Abrufübungen angewendet werden sollten, können Lernende sie nicht adäquat nutzen (Carpenter et al. 2020).

In Lernsituationen mit vielen Freiheitsgraden ist eine weitere Eigenschaft der Lernenden zu berücksichtigen. In vielen Lernsituationen können Lernende selbst entscheiden, ob sie eine gegebene Antwort noch einmal nachbessern wollen oder das Nicht-Gewusste einfach nachschauen, z. B. bei einer Abrufaufgabe, die als Hausaufgabe gegeben wurde. Auch diese selbstgesteuerte Nutzung von erneuten Lerngelegenheiten unterliegt individuellen Eigenschaften der Lernenden. In Roelle und Renkl (2020) beantworteten Lernende u. a. Selbsterklärungsaufgaben mit Abrufanteil. Die Lernenden wurden instruiert, dass sie, wenn sie eine Wissenslücke identifiziert hatten oder wenn sie ihre Antworten überprüfen wollten, eine erneute Lerngelegenheit nutzen sollten. Lernende mit hohem akademischen Selbstkonzept nutzten diese Möglichkeit zu selten, um von der zusätzlichen Lerngelegenheit zu profitieren. Lernende, die ein niedrigeres Selbstkonzept aufwiesen, konnten dadurch ihren Lernerfolg verbessern. Ein hohes akademisches Selbstkonzept scheint die exakte metakognitive Überwachung bzw. die korrekte Regulation zumindest in diesem Fall zu behindern.

Ein weiterer relevanter Faktor in diesem Bereich ist die Prüfungsangst. Lernende, die unter hohem Druck stehen, können weniger von direkten Abrufübungseffekten profitieren als Lernende mit niedrigem wahrgenommenem Druck (Hinze und Rapp 2014). Wenn Abrufaufgaben von hoher Wichtigkeit sind, scheinen Personen, die prüfungsängstlich sind, weniger von Abrufübungen zu profitieren als Nicht-Prüfungsängstliche (Clark et al. 2018). Das Bereitstellen einer erneuten Lerngelegenheit nach dem Abruf kann die Drucksituation der Abrufsituation reduzieren (Núñez-Peña et al. 2015). So können auch Lernende, die normalerweise prüfungsängstlich sind, durch die Hinzunahme indirekter Abrufeffekte profitieren. Sie haben weniger Angst 
und können somit sowohl von direkten wie auch indirekten Abrufübungseffekten profitieren.

Praktische Empfehlungen Die Hinzunahme einer erneuten Lernphase nach dem Abruf ist besonders für Lernende sinnvoll, die in Abrufübungen einen geringen Abruferfolg erzielen, egal ob dies durch geringes Vorwissen oder reduzierte generelle kognitiven Fähigkeiten bedingt ist. Lernende, die geringes Lernstrategiewissen besitzen, sollten besonders unterstützt werden. Diese Lernenden profitieren dann von einer erneuten Lernphase, wenn zusätzliche Regulationsprompts angeboten werden. Das heißt, dass diese Lernenden zusätzlich dazu angeregt werden sollten, spezifische Lernstrategien anzuwenden. Bei Lernenden mit einem besonders hohen akademischen Selbstkonzept scheint es nicht sinnvoll zu sein, die erneute Lernphase optional zur Verfügung zu stellen. Lernende mit hohem Selbstkonzept wählen selten die Möglichkeit, erneut ihre Wissensstrukturen zu verbessern, auch wenn sie davon profitieren könnten. Zuletzt kann man auch testängstlichen Lernenden durch eine erneute Lernphase besonders helfen. Die erneute Lernphase mindert die Bedeutung des Erfolgs bei Tests zur Abrufübung, wodurch auch testängstliche Lernende sich leichter auf relevante Informationen beziehen können. Testängstliche Lernende können so auch den direkten Effekt nutzen.

\section{Fazit}

Das Implementieren einer erneuten Lernphase nach einer Abrufübung kann mehr als nur Feedback für die metakognitive Einschätzung der Lernenden sein. Erneute Lernphasen sind bei bedeutungshaltigem Lernen besonders dann sinnvoll, wenn nicht der Großteil der abgefragten Informationen abgerufen werden konnte. Der Umfang der erneuten Lernphase sollte bei bedeutungshaltigem Lernen die eigentliche Antwort übersteigen. Der Einfluss, den Abrufübungen auf metakognitive Überwachung, Regulation und Motivation haben, ist komplex und sollte abhängig von Lernzielen und den Merkmalen der Lernenden implementiert werden. Dabei kann eine Kombination unterschiedlicher Abrufübungen helfen, multiple Ziele zu erreichen.

Funding Open Access funding enabled and organized by Projekt DEAL.

Open Access Dieser Artikel wird unter der Creative Commons Namensnennung 4.0 International Lizenz veröffentlicht, welche die Nutzung, Vervielfältigung, Bearbeitung, Verbreitung und Wiedergabe in jeglichem Medium und Format erlaubt, sofern Sie den/die ursprünglichen Autor(en) und die Quelle ordnungsgemäß nennen, einen Link zur Creative Commons Lizenz beifügen und angeben, ob Änderungen vorgenommen wurden.

Die in diesem Artikel enthaltenen Bilder und sonstiges Drittmaterial unterliegen ebenfalls der genannten Creative Commons Lizenz, sofern sich aus der Abbildungslegende nichts anderes ergibt. Sofern das betreffende Material nicht unter der genannten Creative Commons Lizenz steht und die betreffende Handlung nicht nach gesetzlichen Vorschriften erlaubt ist, ist für die oben aufgeführten Weiterverwendungen des Materials die Einwilligung des jeweiligen Rechteinhabers einzuholen.

Weitere Details zur Lizenz entnehmen Sie bitte der Lizenzinformation auf http://creativecommons.org/ licenses/by/4.0/deed.de. 


\section{Literatur}

\section{Verwendete Literatur}

Adesope, O. O., Trevisan, D. A., \& Sundararajan, N. (2017). Rethinking the Use of Tests: A Meta-Analysis of Practice Testing. Review of Educational Research. https://doi.org/10.3102/0034654316689306.

Agarwal, P.K. (2019). Retrieval practice \& bloom's taxonomy: do students need fact knowledge before higher order learning? Journal of Educational Psychology, 111(2), 189.

Arnold, K. M., \& McDermott, K. B. (2013). Test-potentiated learning: distinguishing between direct and indirect effects of tests. Journal of Experimental Psychology: Learning, Memory, and Cognition, 39(3), 940.

Arthur C., Graesser Murray, Singer Tom, Trabasso (1994) Constructing inferences during narrative text comprehension. Psychological Review, 101(3), 371-395. https://doi.org/10.1037/0033-295X.101.3. 371.

Athanases, S., \& Achinstein, B. (2003). Focusing New Teachers on Individual and Low Performing Students: The Centrality of Formative Assessment in the Mentor. Teachers College Record, 105(8), $1486-1520$.

Ausubel, D.P. (1968). Educational psychology: a cognitive view. Holt, Rinehart and Winston: New York.

Bandura, A. (1997). The anatomy of stages of change. American journal of health promotion, 12(1), 8-10.

Barenberg, J., \& Dutke, S. (2019). Testing and metacognition: retrieval practise effects on metacognitive monitoring in learning from text. Memory, 27(3), 269-279.

Barnes et al. (1996). The Effects of Knowledge Availability and Knowledge Accessibility on Coherence and Elaborative Inferencing in Children from Six to Fifteen Years of Age. Journal of Experimental Child Psychology, 61, 216-241.

Bender et al. (2021). Seductive details do their damage also in longer learning sessions - When the details are perceived as relevant. https://doi.org/10.1111/jcal.12560.

Biggs, J. (1996). Enhancing teaching through constructive alignment. Higher education, 32(3), 347-364.

Blasiman, R. N., Dunlosky, J., \& Rawson, K. A. (2017). The what, how much, and when of study strategies: comparing intended versus actual study behaviour. Memory, 25(6), 784-792.

de Bruin, A. B.H., Roelle, J., Carpenter, S. K., Baars, M., \& EFG-MRE (2020). Synthesizing cognitive load and self-regulation theory: a theoretical framework and research agenda. Educ Psychol Rev, 32, 903-915.

Bull, S.G., \& Dizney, H.F. (1973). Epistemic-curiosity-arousing prequestions: their effect on long-term retention. Journal of Educational Psychology, 65, 45-49.

Carpenter, S. K. (2009). Cue strength as a moderator of the testing effect: The benefits of elaborative retrieval. Journal of Experimental Psychology: Learning, Memory, and Cognition, 35(6), 1563-1569. https://doi.org/10.1037/a0017021.

Carpenter, S. K., Endres, T., \& Hui, L. (2020). Students' use of retrieval in self-regulated learning: implications for monitoring and regulating effortful learning experiences. Educational Psychology Review, 32(8), 1-26. https://doi.org/10.1007/s10648-020-09562-w.

Carpenter, S. K., Rahman, S., \& Perkins, K. (2018). The effects of prequestions on classroom learning. Journal of Experimental Psychology: Applied, 24(1), 34-42. https://doi.org/10.1037/xap0000145.

Chan, J. C. K. (2009). When does retrieval induce forgetting and when does it inducefacilitation? Implications for retrieval inhibition, testing effect, and text processing.Journal of Memory and Language, 61(2), 153-170. https://doi.org/10.1016/j.jml.2009.04.004.

Chan, J. C. K., Meissner, C. A., \& Davis, S. D. (2018). Retrieval potentiates new learning: A theoretical and meta-analytic review. Psychological Bulletin, 144(11), 1111-1146. https://doi.org/10.1037/ bul0000166.

Chi et al. (1994). Eliciting Self-Explanations Improves Understanding. https://doi.org/10.1016/03640213(94)90016-7.

Cho, K. W., Neely, J. H., Crocco, S. \& Vitrano, D. (2017). Testing enhances both encodingand retrieval for both tested and untested items. Quart. J. Exp. Psychol. 70,1211-1235, https://doi.org/10.1080/ 17470218.2016.1175485.

Clark, D. A., \& Svinicki, M. (2014). The Effect of Retrieval on Post-task Enjoyment of Studying. Educational Psychology Review, 27(1), 51-67. https://doi.org/10.1007/s10648-014-9272-4.

Clark, D. A., Crandall, J. R., \& Robinson, D. H. (2018). Incentives and test anxiety may moderate the effect of retrieval on learning. Learning and Individual Differences, 63, 70-77. https://doi.org/10. 1016/j.lindif.2018.03.001. 
Collins, A. M., \& Loftus, E. F. (1975). A spreading-activation theory of semantic processing. Psychological Review, 82(6), 407-428. https://doi.org/10.1037//0033-295X.82.6.407.

Eitel, A. (2016). How repeated studying and testing affects multimedia learning: Evidence for adaptation to task demands. Learning and Instruction, 41, 70-84. https://doi.org/10.1016/j.learninstruc.2015.10. 003.

Endres, T., \& Renkl, A. (2015). Mechanisms behind the testing effect: an empirical investigation of retrieval practice in meaningful learning. Frontiers in psychology, 6, 1054. https://doi.org/10.3389/fpsyg. 2015.01054.

Endres, T., Kranzdorf, L., Schneider, V., \& Renkl, A. (2020). It matters how to recall-task differences in retrieval practice. Instructional Science, 48(6), 699-728.

Endres, T., Kubik, V., Hahne, F., Beyer, R., \& Renkl, A. (in prep.). The indirect benefits of testing on selfregulated learning with text materials.

Gezer et al. (2021). The Relationship between Formative Assessment and Summative Assessment in Primary Grade Mathematics Classrooms. https://doi.org/10.26822/iejee.2021.220.

Guran, C. N. A., Herweg, N. A., \& Bunzeck, N. (2019). Age-related decreases in the retrieval practice effect directly relate to changes in alpha-beta oscillations. Journal of Neuroscience, 39(22), 4344-4352.

Hausman, H., \& Rhodes, M. G. (2018). When pretesting fails to enhance learning concepts from reading texts. Journal of Experimental Psychology: Applied, 24, 331-346.

Hays, M. J., Kornell, N., \& Bjork, R. A. (2013). When and why a failed test potentiates the effectiveness of subsequent study. Journal of Experimental Psychology: Learning, Memory, and Cognition, 39(1), 290.

Healy, A. F., Jones, M., Lalchandani, L. A., \& Tack, L. A. (2017). Timing of quizzes during learning: effects on motivation and retention. Journal of Experimental Psychology: Applied, 23(2), 128.

Hinze, S. R., \& Rapp, D. N. (2014). Retrieval (sometimes) enhances learning: performance pressure reduces the benefits of retrieval practice. Applied Cognitive Psychology, 28(4), 597-606.

Izawa, C. (1966). Reinforcement-test sequences in paired-associate learning. Psychological Reports, 18(3), 879-919.

Izawa, C. (1970). Optimal potentiating effects and forgetting-prevention effects of tests in paired-associate learning. Journal of Experimental Psychology, 83, 340.

James, K. K., \& Storm, B.C. (2019). Beyond the pretesting effect: what happens to the information that is not pretested? Journal of Experimental Psychology: Applied, 25, 576-587.

Jing, H. G., Szpunar, K. K., \& Schacter, D. L. (2016). Interpolated testing influences focused attention and improves integration of information during a video-recorded lecture. Journal of Experimental Psychology: Applied, 22(3), 305.

Karpicke, J. D., Butler, A. C., \& Roediger III, H. L. (2009). Metacognitive strategies in student learning: do students practise retrieval when they study on their own? Memory, 17(4), 471-479. https://doi.org/ 10.1080/09658210802647009.

King, J. F., Zechmeister, E. B., \& Shaughnessy, J. J. (1980). Judgments of knowing: the influence of retrieval practice. The American Journal of Psychology, 93(2), 329-343. https://doi.org/10.2307/1422236.

Kingston, N., \& Nash, B. (2011). Formative assessment: a meta-analysis and a call for research. Educational measurement: Issues and practice, 30(4), 28-37.

Kintsch, W. (1988). The role of knowledge in discourse comprehension: A construction-integration model. Psychological Review, 95(2), 163-182. https://doi.org/10.1037/0033-295X.95.2.163.

Koedinger, K. R., \& Mclaughlin, E. A. (2010). A quasi-experimental evaluation of an on-line formative assessment and tutoring system. Carnegie Mellon University. Neil T. Heffernan. Worcester Polytechnic Institute. https://doi.org/10.2190/EC.43.4.d.

Koriat, A. (2007). Metacognition and consciousness. In P.D. Zelazo, M. Moscovitch \& E. Thompson (Hrsg.), The Cambridge handbook of consciousness (S. 289-325). Cambridge: Cambridge University Press.

Koriat, A. (2012). The self-consistency model of subjective confidence. Psychological Review, 119(1), 80.

Kuhbandner, C., \& Emmerdinger, K. J. (2019). Do students really prefer repeated rereading over testing when studying textbooks? A reexamination. Memory, 27(7), 952-961. https://doi.org/10.1080/ 09658211.2019 .1610177$.

Leber, J., Renkl, A., Nückles, M., \& Wäschle, K. (2018). When the type of assessment counteracts teaching for understanding. Learning: Research and Practice, 4(2), 161-179.

Lechuga, M.T., Moreno, V., Pelegrina, S., Gómez-Ariza, C. J., \& Bajo, M. T. (2006). Age differences in memory control: evidence from updating and retrieval-practice tasks. Acta Psychologica, 123(3), 279-298. 
Leeming, F. C. (2002). The exam-a-day procedure improves performance in psychology classes. Teaching of Psychology, 29(3), 210-212.

Lindner, M. A., Strobel, B., \& Köller, O. (2015). Multiple-Choice-Prüfungen an Hochschulen? Ein Literaturüberblick und Plädoyer für mehr praxisorientierte Forschung. Zeitschrift für Pädagogische Psychologie, 29, 133-149. https://doi.org/10.1024/1010-0652/a000156.

Little, J. L., \& McDaniel, M. A. (2014). Metamemory monitoring and control followingretrieval practice for text. Memory and Cognition, 43(1), 85-98. https://doi.org/10.3758/s13421-014-0453-7

Lovelace, E. A. (1984). Metamemory: Monitoring future recallability during study. Journal of Experimental Psychology: Learning, Memory, and Cognition, 10(4), 756-766. https://doi.org/10.1037/02787393.10.4.756

Lyle, K. B., \& Crawford, N. A. (2011). Retrieving essential material at the end of lectures improves performance on statistics exams. Teaching of Psychology, 38(2), 94-97.

Mayer, R. E. (1997). From novice to expert. In Handbook of human-computer interaction (S. 781-795). North-Holland.

McCrudden, M. T., Schraw, G., \& Kambe, G. (2005). The effect of relevance instructions on reading time and learning. Journal of Educational Psychology, 97, 88-102.

McDaniel, M. A., \& Little, J. L. (2019). Multiple-choice and short-answer quizzing on equal footing in the classroom: Potential indirect effects of testing. In J. Dunlosky \& K. A. Rawson (Hrsg.), The Cambridge handbook of cognition and education (S. 480-499). Cambridge University Press. https:// doi.org/10.1017/9781108235631.020.

McNamara, D. S., \& Kintsch, W. (1996). Learning from texts: Effects of prior knowledge and text coherence. Discourse Processes, 22(3), 247-288. https://doi.org/10.1080/01638539609544975.

Memory, D. M. (1983). Main idea prequestions as adjunct aids with good and low-average middle grade readers. Journal of Reading Behavior, 15, 37-48.

Metcalfe, J., \& Kornell, N. (2005). A Region of Proximal Learning model of study time allocation. Journal of memory and language, 52(4), 463-477. https://doi.org/10.1016/j.jml.2004.12.001.

Moss, C. M., \& Brookhart, S. M. (2019). Advancing formative assessment in every classroom: A guide for instructional leaders. ASCD.

Müller, N. M., \& Seufert, T. (2018). Effects of self-regulation prompts in hypermedia learning on learning performance and self-efficacy. Learning and Instruction, 58, 1-11.

Narciss, S. (2020). Feedbackstrategien für interaktive Lernaufgaben. In: Niegemann H., Weinberger A. (eds) Handbuch Bildungstechnologie. Berlin, Heidelberg: Springer. https://doi.org/10.1007/978-3662-54368-9_35.

Nückles, M., Roelle, J., Glogger-Frey, I., Waldeyer, J., \& Renkl, A. (2020). The self-regulation-view in writing-to-learn: using journal writing to optimize cognitive load in self-regulated learning. Educational Psychology Review, 32, 1089-1126. https://doi.org/10.1007/s10648-020-09541-1.

Núñez-Peña, M. I., Bono, R., \& Suárez-Pellicioni, M. (2015). Feedback on students' performance: a possible way of reducing the negative effect of math anxiety in higher education. International Journal of Educational Research, 70, 80-87.

Pan, S.C., \& Rickard, T. C. (2018). Transfer of test-enhanced learning: meta-analytic review and synthesis. Psychological Bulletin, 144(7), 710.

Pan, S.C., \& Sana, F. (2021). Pretesting versus posttesting: comparing the pedagogical benefits of errorful generation and retrieval practice. Journal of Experimental Psychology: Applied, 27(2), 237-257. https://doi.org/10.1037/xap0000345.

Pastötter, B., \& Bäuml, K.-H. T. (2014). Retrieval practice enhances new learning: The forward effect of testing. Frontiers in Psychology, 5, Article 286.

Pintrich, P. R. (2002). The role of metacognitive knowledge in learning, teaching, and assessing. Theory into practice, 41(4), 219-225.

Pressley, M., Tanenbaum, R., McDaniel, M.A., \& Wood, E. (1990). What happens when university students try to answer prequestions that accompany textbook material? Contemporary Educational Psychology, 15, 27-35.

Renkl, A., \& Stern, E. (1994). Die Bedeutung von kognitiven Eingangsvoraussetzungen und. Zeitschrift für pädagogische Psychologie, 8(1), 27-39.

Renkl, A., Skuballa, I.T., Schwonke, R., Harr, N., \& Leber, J. (2015). The effects of rapid assessments and adaptive restudy prompts in multimedia learning. Journal of Educational Technology \& Society, 18(4), 185-198.

Renkl, A. (2015). Wissenserwerb. In E. Wild \& J. Möller (Hrsg.), Pädagogische Psychologie (2. überarbeitete Auflage) (S. 3-24). Berlin: Springer. 
Rickards, J.P. (1976). Interaction of position and conceptual level of adjunct questions on immediate and delayed retention of text. Journal of Educational Psychology, 68, 210-217.

Rivers, M.L. (2020). Metacognition about practice testing: a review of learners' beliefs, monitoring, and control of test-enhanced learning. Educational Psychology Review. https://doi.org/10.1007/s10648020-09578-2.

Roediger III, H.L., \& Butler, A.C. (2011). The critical role of retrieval practice in long-term retention. Trends in cognitive sciences, 15(1), 20-27.

Roediger III, H. L., \& Karpicke, J.D. (2006). The power of testing memory: basic research and implications for educational practice. Perspectives on psychological science, 1(3), 181-210.

Roediger, H. L. III, Putnam, A. L., \& Smith, M. A. (2011). Ten benefits of testing and their applications to educational practice. In J. P. Mestre \& B. H. Ross (Hrsg.), The psychology of learning and motivation: Cognition in education (S 1-36). Elsevier Academic Press. https://doi.org/10.1016/B978-0-12387691-1.00001-6.

Roelle, J., \& Renkl, A. (2020). Does an option to review instructional explanations enhance example-based learning? It depends on learners' academic self-concept. Journal of Educational Psychology, 112(1), 131.

Rotgans, \& Schmidt. (2011). Situational interest and academic achievement in the active-learning classroom. https://doi.org/10.1016/j.learninstruc.2009.11.001.

Rothkopf, E.Z., \& Billington, M.J. (1979). Goal-guided learning from text: inferring a descriptive processing model from inspection times and eye movements. Journal of Educational Psychology, 71, 310-327.

Rowland, C. A. (2014). The effect of testing versus restudy on retention: a meta-analytic review of the testing effect. Psychological Bulletin, 140(6), 1432.

Schmidt, H.G., \& Rotgans, J.I. (2021). Epistemic curiosity and situational interest: distant cousins or identical twins? Educational Psychology Review, 33, 325-352. https://doi.org/10.1007/s10648-02009539-9.

Schunk, D.H. (1985). Self-efficacy and classroom learning. Psychology in the Schools, 22(2), 208-223.

Shaughnessy, J. J., \& Zechmeister, E. B. (1992). Memory-monitoring accuracy as influenced by the distribution of retrieval practice. Bulletin of the Psychonomic Society, 30(2), 125-128. https://doi.org/10. 3758/BF03330416.

Shute, V.J. (2008). Focus on formative feedback. Review of educational research, 78(1), 153-189. https:// doi.org/10.3102/0034654307313795.

Souvignier, E., \& Hasselhorn, M. (2018). Formatives Assessment. Zeitschrift für Erziehungswissenschaften, 21, 693-996.

Spitzer, H.F. (1939). Studies in retention. Journal of Educational Psychology, 30(9), 641.

St. Hilaire, K. J., Carpenter, S. K., \& Jennings, J. M. (2019). Using prequestions to enhance learning from reading passages: the roles of question type and structure building ability. Memory, 27(9), 1204-1213.

Szpunar, K. K., Khan, N. Y., \& Schacter, D.L. (2013). Interpolated memory tests reduce mind wandering and improve learning of online lectures. Proceedings of the National Academy of Sciences, 110(16), 6313-6317.

Tullis, J. G., \& Maddox, G. B. (2020). Self-reported use of retrieval practice varies across age and domain. Metacognition and Learning, 15, 129-154.

Tullis, J.G., Finley, J.R., \& Benjamin, A.S. (2013). Metacognition of the testing effect: guiding learners to predict the benefits of retrieval. Memory \& cognition, 41(3), 429-442.

Van Gog, T., \& Sweller, J. (2015). Not new, but nearly forgotten: the testing effect decreases or even disappears as the complexity of learning materials increases. Educational Psychology Review, 27(2), 247-264.

Wang et al. (2014). Developing an assessment-centered e-Learning system for improving student learning effectiveness. Computers \& Education, 73, 189-203.

Winne, P.H. (2011). A cognitive and metacognitive analysis of self-regulated learning. In B. J. Zimmerman \& D. H. Schunk (Hrsg.), Handbook of self-regulation of learning and performance (S. 15-32). Routledge/Taylor \& Francis Group.

Winne, P.H., \& Hadwin, A.F. (1998). Studying as self-regulated learning. In D. J. Hacker, J. Dunlosky, \& A.C. Graesser (Hrsg.), Metacognition in educational theory and practice (S. 277-304). Lawrence Erlbaum Associates Publishers.

Wissman, K. T., Rawson, K. A., \& Pyc, M.A. (2011). The interim test effect: testing prior material can facilitate the learning of new material. Psychonomic Bulletin \& Review, 18(6), 1140-1147. https:// doi.org/10.3758/s13423-011-0140-7. 
Yang, C., Potts, R., \& Shanks, D. R. (2018). Enhancing learning and retrieval of new information: a review of the forward testing effect. NPJ science of learning, 3(1), 1-9.

Yang, C., Luo, L., Vadillo, M. A., Yu, R., \& Shanks, D. R. (2021). Testing (quizzing) boosts classroom learning: A systematic and meta-analytic review. Psychological Bulletin, 147(4), 399-435. https:// doi.org/10.1037/bu10000309.

Yue, C.L., Soderstrom, N.C., \& Bjork, E.L. (2015). Partial testing can potentiate learning of tested and untested material from multimedia lessons. Journal of Educational Psychology, 107(4), 991.

Zimmerman, B.J. (1990). Self-regulated learning and academic achievement: An overview. Educational psychologist, 25(1), 3-17. https://doi.org/10.3102/0002831207312909.

\section{Weiterführende Literatur}

Daniel A., Clark Jason R., Crandall Daniel H., Robinson (2018) Incentives and test anxiety may moderate the effect of retrieval on learning. Learning and Individual Differences 6370-77 https://doi.org/10. 1016/j.lindif.2018.03.001.

Shana K., Carpenter Shuhebur, Rahman Kyle, Perkins (2018) The effects of prequestions on classroom learning. Journal of Experimental Psychology: Applied, 24(1), 34-42. https://doi.org/10.1037/ xap0000145. 